\author{
ANNALES \\ POLONICI MATHEMATICI \\ XXIV (1971)
}

\title{
On a linear functional equation of second order
}

\author{
by K. BADURA (Katowice)
}

In the present paper we shall extend some results from [1] concerning the solution of the linear functional equation of the second order

$$
\varphi\left[f^{2}(x)\right]+A(x) \varphi[f(x)]+B(x) \varphi(x)=F(x),
$$

where $\varphi(x)$ is the required function and $f(x), F(x), A(x), B(x)$ are given functions. We shall denote by $f^{k}(x)$ the $k$-th iteration of the function $f(x)$, i.e. we put

$$
\begin{gathered}
f^{0}(x)=x, \quad f^{k+1}(x)=f\left[f^{k}(x)\right], \\
f^{-k-1}(x)=f^{-1}\left[f^{-k}(x)\right], \quad k=0,1,2, \ldots
\end{gathered}
$$

According to the results obtained by Kuczma [2] concerning the reduction of the order, the investigation of equation (1) may be replaced by the study of suitable equations of lower orders.

Let $\lambda(x)$ be a solution of the functional equation:

$$
\lambda[f(x)] \lambda(x)+A(x) \lambda(x)+B(x)=0,
$$

and let. us put:

$$
\mu(x) \stackrel{\text { df }}{=}-A(x)-\lambda[f(x)]
$$

LEMMA I. Equation (1) is equivalent to the system of equations of the first order with the unknown functions $\varphi(x)$ and $\psi(x)$ :

$$
\begin{cases}\text { (i) } & \varphi[f(x)]-\lambda(x) \varphi(x)=\psi(x), \\ \text { (ii) } \quad \psi[f(x)]-\mu(x) \psi(x) & =F^{\prime}(x) .\end{cases}
$$

The proof of this lemma may be found in [2], p. 260.

The next lemma will concern the solution of the linear equation of the first order:

$$
\varphi[f(x)]-\lambda(x) \varphi(x)=G(x) .
$$


Let us make the following assumption about the function $f(x)$ :

Assumption $A-f(x)$ is a real-valued function of the real variable $x_{\text {r }}$ continuous and strictly increasing in an interval $(a, b)$, and $f(b)=b$, $f(x)>x$ in $(a, b)$.

According to the notation used in [2] we have $f_{\epsilon} \boldsymbol{R}_{b}^{0}[(a, b)]$.

LEMmA II. Let $f(x)$ satisfy assumption $\mathrm{A}$, and let $\lambda(x)$ and $G(x)$ be complex-valued functions of the real variable $x$, continuous in the interval. $(a, b)$. Then equation (5) possesses in the open interval $(a, b)$ a continuous solution depending on an arbitrary function. More precisely, for any $x_{0} \epsilon(a, b)$, and for an arbitrary function $\bar{\varphi}(x)$ continuous in the interval $\left\langle x_{0}, x_{1}\right)$ and fulfilling the condition

$$
\lim _{x \rightarrow x_{1}^{-}} \bar{\varphi}(x)=G\left(x_{0}\right)+\lambda\left(x_{0}\right) \bar{\varphi}\left(x_{0}\right)
$$

there exists exactly one solution $\varphi(x)$ of equation (5) continuous in the open interval $(a, b)$ and such that $\varphi(x)=\bar{\varphi}(x)$ in $\left\langle x_{0}, x_{1}\right)$. This solution is given by the formula

$$
\varphi(x)= \begin{cases}\bar{\varphi}(x), & x \epsilon\left\langle x_{0}, x_{1}\right), \\ G\left[f^{-1}(x)\right]+\lambda\left[f^{-1}(x)\right] \varphi\left[f^{-1}(x)\right], & x \epsilon\left\langle x_{n}, x_{n+1}\right), \\ \frac{\varphi[f(x)]-G(x)}{\lambda(x)}, & x \epsilon\left\langle x_{-n}, x_{-n+1}\right), \\ n=1,2, \ldots\end{cases}
$$

where $x_{n} \stackrel{d f}{=} f^{n}\left(x_{0}\right), n= \pm 1, \pm 2, \ldots$

If, moreover, the functions $\lambda(x)$ and $G(x)$ are continuous in the interval $(a, b)$, then:

(a) In the case $|\lambda(b)|>1$ equation (5) possesses exactly one solution continuous in the interval $(a, b)$.

This solution has the form

$$
\varphi(x)=-\sum_{v=0}^{\infty} \frac{G\left[f^{\nu}(x)\right]}{\prod_{i=0}^{\nu} \lambda\left[f^{i}(x)\right]}
$$

(b) In the case $|\lambda(b)|<1$ every solution of equation (1) in the interval $(a, b)$ continuous in $(a, b)$ is also continuous in the interval $(a, b\rangle$; in view of the first part of the theorem case (b) says that equation (5) possesses in the interval $(a, b)$ a continuous solution depending on an arbitrary function.

Lemma II is an immediate consequence of the results found in book [2], chapter II. 
The following fundamental theorem concerning continuous solutions of equation (1) has been proved by Kordylewski and Kuczma [1]. Their proof, however, contains an inaccuracy. In the proof given below this inaccuracy has been removed and we push forward the investigation of the number of continuous solutions of equation (1) in case II, $3^{\circ}$, below.

Let $d_{1}$ and $d_{2}$ be roots of the equation

$$
d^{2}+A(b) d+B(b)=0 .
$$

THEOREM 1. Let the function $f(x)$ fulfil asumption $\mathrm{A}$, and let $\boldsymbol{A}(x)$, $B(x)$ and $F(x)$ be complex-valued functions of the real variable $x$, continuous in the interval $(a, b\rangle, B(x) \neq 0$ in $(a, b)$. Under these hypothesis:

I. Equation (1) possesses in the open interval $(a, b)$ a continuous solution depending on an arbitrary function. More precisely, for any $x_{0} \in(a, b)$ and for an arbitrary function $\varphi_{0}(x)$ continuous in $\left\langle x_{0}, x_{2}\right)$ and fulfilling the condition:

$$
\lim _{x \rightarrow x_{2}^{-}} \varphi_{0}(x)=F^{\prime}\left(x_{0}\right)-A\left(x_{0}\right) \varphi_{0}\left[f\left(x_{0}\right)\right]-B\left(x_{0}\right) \varphi_{0}\left(x_{0}\right)
$$

there exist exactly one solution $\varphi(x)$ of equation (1) continuous in $(a, b)$. and such that $\varphi(x)=\varphi_{0}(x)$ for $x \epsilon\left\langle x_{0}, x_{2}\right)$.

II. Let us suppose, moreover, that $\left|d_{1}\right| \neq\left|d_{2}\right|$. Then:

$1^{\circ}$ If $\left|d_{1}\right|>1$ and $\left|d_{2}\right|>1$, then equation (1) has exactly one solution continuous in the interval $(a, b\rangle$.

$2^{\circ}$ If $\left|d_{1}\right|<1$ and $\left|d_{2}\right|<1$, then every solution of equation (1) in $(a, b\rangle$. that is continuous in the open interval $(a, b)$ is also continuous in the interval $(a, b)$.

$3^{\circ}$ If $\left|d_{1}\right|>1$ and $\left|d_{2}\right|<1$, then equation (1) possesses infinitely many solutions that are continuous in the interval $(a, b>$.

Proof. It has been proved by Kuczma and Vopénka [3] under the hypotheses of the present theorem that equation (2) has a continuous solution in the interval $(a, b)$ fulfilling the conditions

$$
\lambda(x) \neq 0, \quad \lambda(x) \neq-A\left[f^{-1}(x)\right] \quad \text { for } x \in(a, b) .
$$

Hence, by Lemma I, we infer that equation (1) is equivalent to the system of equations (4), where the functions $\lambda(x)$ and $\mu(x)$ are continuous and different from zero in $(a, b)$. It follows from Lemma II that equation (ii) has in the interval $(a, b)$ a continuous solution depending on an arbitrary function. This solution, as the right-hand side of equation (4), (i), gives rise to a new family of continuous solutions depending on an arbitrary function. Simultaneously these are solutions of equation (1).

We shall show that this family depends on an arbitrary function $\varphi_{0}(x)$ defined and continuous in the interval $\left\langle x_{0}, x_{2}\right)$ and fulfilling condition (10). Indeed, let $\varphi(x)=\varphi_{0}(x)$ for $x \epsilon\left\langle x_{0}, x_{2}\right)$, where $\varphi_{0}(x)$ is this arbi- 
trary function. From equation (i) of system (4) we then obtain a function $\psi_{0}(x)$ determined and continuous in the interval $\left\langle x_{0}, x_{1}\right)$. We have

$$
\begin{aligned}
\lim _{x \rightarrow x_{1}^{-}} \psi_{0}(x)= & \lim _{x \rightarrow x_{1}^{-}} \varphi_{0}[f(x)]-\lambda(x) \varphi_{0}(x) \\
= & \lim _{x \rightarrow x_{2}^{-}} \varphi_{0}(x)-\lambda\left[f\left(x_{0}\right)\right] \varphi_{0}\left[f\left(x_{0}\right)\right] \\
= & F\left(x_{0}\right)-A\left(x_{0}\right) \varphi_{0}\left[f\left(x_{0}\right)\right]-B\left(x_{0}\right) \varphi_{0}\left(x_{0}\right)-\lambda\left[f\left(x_{0}\right)\right] \varphi_{0}\left[f\left(x_{0}\right)\right] \\
= & F\left(x_{0}\right)-A\left(x_{0}\right) \varphi_{0}\left[f\left(x_{0}\right)\right]+\left\{A\left(x_{0}\right) \lambda\left(x_{0}\right)+\lambda\left[f\left(x_{0}\right)\right] \lambda\left(x_{0}\right)\right\} \varphi_{0}\left(x_{0}\right)- \\
& -\lambda\left[f\left(x_{0}\right)\right] \varphi_{0}\left[f\left(x_{0}\right)\right] \\
= & F\left(x_{0}\right)+\left(-A\left(x_{0}\right)-\lambda\left[f\left(x_{0}\right)\right]\right)\left(\varphi_{0}\left[f\left(x_{0}\right)\right]-\lambda\left(x_{0}\right) \varphi_{0}\left(x_{0}\right)\right) \\
= & F\left(x_{0}\right)+\mu\left(x_{0}\right) \psi_{0}\left(x_{0}\right) .
\end{aligned}
$$

Hence and from Lemma II it follows that equation (ii) of system (4) determines the function $\psi(x)$ in the whole interval $(a, b)$. Having defined the function $\psi(x)$ in this manner, we return to equation (i), which also has a solution depending on an arbitrary function $\bar{\varphi}(x)$ - determined and continuous in the interval $\left\langle x_{0}, x_{1}\right)$ and fulfilling the condition

$$
\lim _{x \rightarrow x_{1}^{-}} \bar{\varphi}(x)=\lambda\left(x_{0}\right) \varphi\left(x_{0}\right)+\psi\left(x_{0}\right) .
$$

If we put $\bar{\varphi}(x)=\varphi_{0}(x)$ for $\left\langle x_{0}, x_{1}\right)$, then this condition obviously follows from the continuity of the function $\varphi_{0}(x)$ in the interval $\left\langle x_{0}, x_{2}\right)$. Thus equation (i) determines the function $\varphi(x)$ in the whole interval $(a, b)$. The uniqueness follows also from Lemma .II.

II. If $\left|d_{1}\right| \neq\left|d_{2}\right|$, then equation (2) has a continuous solution in $(a, b)$ fulfilling conditions (11). This theorem is to be found in [3]. But the proof of this theorem was carried out under the following essential hypothesis:

$$
\left|d_{1}\right|>\left|d_{2}\right|, \quad \text { where } d_{1}=\lambda(b) .
$$

Putting $x=b$ in equation (2), we infer by (3) that $\lambda(b)$ and $\mu(b)$ are the roots of equation (9). Thus, if we want to use that theorem, we must complete the hypotheses by condition (12). Then equation (1) is equivalent to the system of equations (4), where the functions $\lambda(x), \mu(x)$ are continuous and different from zero in $(a, b)$; also $\mu(b) \neq 0$ and $\lambda(b) \neq 0$, but this follows from the fact that $B(b) \neq 0$ in equation (9). Now, we shall deal with three possible cases.

$1^{\circ}$ Since $|\lambda(b)|>1$ and $|\mu(b)|>1$, solving system (4) we may use . Lemma II - part (a). In virtue of this lemma equation (1) possesses 
exactly one continuous solution in $(a, b\rangle$. This solutions is given by the formula

$$
\varphi(x)=-\sum_{v=0}^{\infty} \frac{\psi\left[f^{\nu}(x)\right]}{\prod_{i=0}^{v} \lambda\left[f^{i}(x)\right]}, \quad \text { where } \psi(x)=-\sum_{v=0}^{\infty} \frac{F\left[f^{\nu}(x)\right]}{\prod_{i=0}^{v} \mu\left[f^{i}(x)\right]}
$$

$2^{\circ}$ By an analogical argument we find that every solution of equation (1) in $(a, b\rangle$ which is continuous in the open interval $(a, b)$ is also continuous in the interval $(a, b)$.

In view of the first part of the present theorem this case says that equation (1) has a continuous solution in $(a, b)$ depending on an arbitrary function $\varphi_{0}(x)$ defined and continuous in the interval of the form $\left\langle x_{0}, x_{2}\right)$ and fulfilling condition (10).

$3^{\circ}$ As the third case the authors of [1] considered the situation $\left|d_{1}\right|<1$ and $\left|d_{2}\right|>1$, where $d_{1}=\lambda(b), d_{2}=\mu(b)$. Unfortunately, in this case we cannot refer to a theorem from [3] concerning the continuous solutions of equation (2) in the interval $(a, b\rangle$ for the condition that $|\lambda(b)|$ is greater than $|\mu(b)|$ is not fulfilled.

Thus it is necessary to consider the case where $\left|d_{1}\right|>1$ and $\left|d_{2}\right|<1$, as it is stated in the assumptions of the theorem. However, in this case the theorem results also immediately from Lemma II.

In the sequel we should like to obtain additional informations about this infinite family of solutions. On the ground of the equivalence of equation (1) and system (4), we shall deal as before with the system.

Let us denote by $\psi_{a}(x)$ the solution of equation (ii) continuous in $(a, b>$ and depending on an arbitrary function; $a$ is an index connected with the function $\bar{\varphi}(x)$ defined and continuous in the interval of the form $\left\langle x_{0}, x_{1}\right\rangle$ generating this solution.

For every $a$ equation (i) has exactly one solution $\varphi_{a}(x)$ continuous $\mathrm{n}\langle a, b\rangle$; it is given by the formula

$$
\varphi_{a}(x)=-\sum_{v=0}^{\infty} \frac{\psi_{a}\left[f^{\nu}(x)\right]}{\prod_{i=\mathbf{0}}^{p} \lambda\left[f^{i}(x)\right]}
$$

Thus we obtain a family of solutions of equation (1) continuous in the interval $(a, b>$ and depending in some manner by relation (13) on an arbitrary function. But is it the dependence of the solution of the functional equation on an arbitrary function according to the definition in $[2]$, p. 45.

Let us try to transform formula (13) so as to eliminate the dependence of $\varphi_{a}(x)$ on the value of the function $\psi_{a}\left[f^{\nu}(x)\right], v=1,2, \ldots$ Using repeatedly the formula

$$
\psi[f(x)]=\mu(x) \psi(x)+F(x)
$$

Annales Polonicl Mathematicl XXIV 
derived from equation (ii), one can prove by induction the formula

$$
\begin{array}{r}
\frac{\psi\left[f^{n}(x)\right]}{\mu(x) \ldots \mu\left[f^{n-1}(x)\right]}=\psi(x)+\frac{F(x)}{\mu(x)}+\frac{F[f(x)]}{\mu(x) \mu[f(x)]}+\ldots+ \\
+\frac{F\left[f^{n-1}(x)\right]}{\mu(x) \ldots \mu\left[f^{n-1}(x)\right]} .
\end{array}
$$

Putting

$$
M_{n}(x)=\prod_{i=0}^{n-1} \mu\left[f^{i}(x)\right], \quad L_{n}(x)=\prod_{i=0}^{n-1} \lambda\left[f^{i}(x)\right]
$$

we can write the last relation in the form

$$
\frac{\psi\left[f^{n}(x)\right]}{M_{n}(x)}=\psi(x)+\sum_{i=0}^{n-1} \frac{F\left[f^{i}(x)\right]}{M_{i+1}(x)} .
$$

Hence

$$
\frac{\psi\left[f^{n}(x)\right]}{\prod_{i=0}^{n} \lambda\left[f^{i}(x)\right]}=\frac{\psi_{a}\left[f^{v}(x)\right]}{L_{v+1}(x)}=\psi_{a}(x) \frac{M_{v}(x)}{L_{v+1}(x)}+\frac{M_{v}(x)}{L_{v+1}(x)} \sum_{i=0}^{v-1} \frac{F\left[f^{i}(x)\right]}{M_{i+1}(x)} .
$$

In view of the above representation formula (13) takes the form

$$
\varphi_{a}(x)=-\frac{\psi_{a}(x)}{\lambda(x)}-\sum_{\nu=1}^{\infty}\left\{\psi_{a}(x) \frac{M_{v}(x)}{L_{v+1}(x)}+\frac{M_{v}(x)}{L_{v+1}(x)} \sum_{i=0}^{p-1} \frac{F\left[f^{i}(x)\right]}{M_{i+1}(x)}\right\},
$$

or the equivalent one

$$
\begin{aligned}
\varphi_{a}(x)=-\psi_{a}(x)\left(\frac{1}{\lambda(x)}+\sum_{v=1}^{\infty} \frac{M_{v}(x)}{L_{v+1}(x)}\right)- & \\
& \quad-\sum_{v=1}^{\infty} \frac{M_{v}(x)}{L_{v+1}(x)} \cdot \sum_{i=0}^{\nu-1} \frac{F\left[f^{i}(x)\right]}{M_{i+1}(x)} .
\end{aligned}
$$

The left-hand side of this equality is a continuous function, but we cannot infer hence anything about the convergence of the series from the right-hand side. Let us consider the series

$$
\sum_{v=1}^{\infty} \frac{M_{v}(x)}{L_{v+1}(x)} \text { in }(a, b) .
$$

We shall look for a numerical majorant of this series. It has been proved in [2] that

$$
\lim _{k \rightarrow \infty} f^{k}(x)=b .
$$


In view of the continuity of the function $\lambda(x)$ in $(a, b>$ and by the fact that $|\lambda(b)|>1$ it follows that for an arbitrary number $\varepsilon>0$ we can find an index $K_{1}$ such that for $k>K_{1}$ and $x \in\langle a+\varepsilon, b\rangle$ we have $\left|\lambda\left[f^{k}(x)\right]\right|$ $>R>1$. Similary, since $|\mu(b)|<1$, we have $\left|\mu\left[f^{k}(x)\right]\right|<\varrho<1$ for $k>K_{2}$.

Putting $K=\max \left(K_{1}, K_{2}\right)$ we have for $x \epsilon\langle a+\varepsilon, b\rangle$ and $k>K$

$$
\left|\lambda\left[f^{k}(x)\right]\right|>R>1, \quad\left|\mu\left[f^{k}(x)\right]\right|<\varrho<1 .
$$
series

Moreover, $|\mu(x)| \leqslant N,|\lambda(x)| \geqslant L>0$ for $x \epsilon\langle a+\varepsilon, b\rangle$. Thus the

$$
\sum_{\nu=1}^{K} \frac{N^{\nu}}{L^{\nu+1}}+\sum_{\nu=K+1}^{\infty} \frac{1}{R}\left(\frac{N}{L}\right)^{K+1}\left(\frac{\varrho}{R}\right)^{\nu-K-1}
$$

is the numerical majorant of series (15).

Consequently, series (15) uniformly converges in $\langle a+\varepsilon, b\rangle$ for every $\varepsilon>0$, and thus its sum is a continuous function in $(a, b\rangle$.

Let us put

$$
h(x) \stackrel{\mathrm{df}}{=}-\left(\frac{1}{\lambda(x)}+\sum_{p=1}^{\infty} \frac{M_{p}(x)}{L_{p+1}(x)}\right) .
$$

The function $h(x)$ is continuous in the interval $(a, b)$. Now, the convergence of the second series in the right-hand side of relation (14) follows from the above considerations. Let

$$
g(x) \stackrel{\text { de }}{=}-\sum_{\nu=1}^{\infty} \frac{M_{v}(x)}{L_{v+1}(x)} \cdot \sum_{i=0}^{\nu-1} \frac{F\left[f^{i}(x)\right]}{M_{i+1}(x)}
$$

The function $g(x)$ is also continuous in the interval $(a, b>$. Thus we finally infer that the continuous solution of equation (1) in $(a, b>$ is given by the formula

$$
\varphi_{a}(x)=\psi_{a}(x) h(x)+g(x),
$$

where $\psi_{a}(x)$ is the continuous solution of equation (ii) in $(a, b>$ depending on an arbitrary function, i.e., in view of (7) and (6)

$$
\psi_{a}(x)= \begin{cases}\bar{\psi}_{a}(x), & x \in\left\langle x_{0}, x_{1}\right), \\ F\left[f^{-1}(x)\right]+\mu\left[f^{-1}(x)\right] \psi_{a}\left[f^{-1}(x)\right], & x \epsilon\left\langle x_{n}, x_{n+1}\right), \\ \frac{\psi_{a}[f(x)]-F(x)}{\mu(x)}, & x \in\left\langle x_{-n}, x_{-n+1}\right), n=1,2, \ldots,\end{cases}
$$


where $\bar{\psi}_{a}(x)$ is an arbitrary function continuous is the interval $\left\langle x_{0}, x_{1}\right)$ and fulfilling the condition

$$
\lim _{x \rightarrow x_{1}^{-}} \vec{\psi}_{a}(x)=F\left(x_{0}\right)+\mu\left(x_{0}\right) \bar{\psi}_{a}\left(x_{0}\right) .
$$

Now, let us calculate the value of the function $h(x)$ at the point $\boldsymbol{x}=\boldsymbol{b}$ :

$$
\begin{aligned}
h(b) & =-\left(\frac{1}{\lambda(b)}+\sum_{v=1}^{\infty} \frac{M_{v}(b)}{L_{v+1}(b)}\right)=-\left(\frac{1}{\lambda(b)}+\sum_{v=1}^{\infty} \frac{\mu(b)^{v}}{\lambda(b)^{v+1}}\right) \\
& =-\frac{1}{\lambda(b)}\left(\begin{array}{c}
1+\frac{\mu(b)}{\lambda(b)} \\
1-\frac{\mu(b)}{\lambda(b)}
\end{array}\right)=-\frac{1}{\lambda(b)}\left(1+\frac{\mu(b)}{\lambda(b)} \frac{\lambda(b)}{\lambda(b)-\mu(b)}\right) \\
& =-\frac{1}{\lambda(b)} \cdot \frac{\lambda(b)}{\lambda(b)-\mu(b)}=\frac{1}{\mu(b)-\lambda(b)} .
\end{aligned}
$$

Since $\mu(b) \neq \lambda(b)$ by hypothesis, $h(b)$ is defined and different from zero. Moreover, $h(x)$ is continuous in the interval $(a, b\rangle$; so there exists a positive number $\eta$ such that

$$
h(x) \neq 0 \quad \text { for } x \epsilon(b-\eta, b>\text {. }
$$

Making use of these facts, we formulate the theorem in case $3^{\circ}$ as an independent theorem.

ThEOREM 2. If the functions $f(x), A(x), B(x), F(x)$ fulfil the $h y$ potheses of Theorem 1 , and $d_{1}, d_{2}$ are roots of equation (9) such that

$$
\left|d_{1}\right|>1, \quad\left|d_{2}\right|<1, \quad \text { where } d_{1}=\lambda(b),
$$

then equation (1) possesses in the interval $(a, b>a$ continuous solution depending on an arbitrary function given in the interval of the form $\left\langle x_{0}, x_{1}\right)$.

Proof. Let the function $\bar{\varphi}_{a}(x)$ be defined and continuous in the interval of the form $\left\langle x_{0}, x_{1}\right\rangle$, where $x_{0}$ is an arbitrary point belonging to the interval $(b-\eta, b\rangle$, and let $\bar{\varphi}_{a}(x)$ fulfil the condition

$$
\lim _{x \rightarrow x_{1}^{-}} \bar{\varphi}_{a}(x)=\left[F\left(x_{0}\right)+\mu\left(x_{0}\right) \frac{\bar{\varphi}_{a}\left(x_{0}\right)-g\left(x_{0}\right)}{h\left(x_{0}\right)}\right] \cdot h\left(x_{1}\right)+g\left(x_{1}\right) .
$$

To this function one can assign the function

$$
\bar{\varphi}_{a}(x)=\frac{\bar{\varphi}_{a}(x)-g(x)}{h(x)}
$$

fulfilling condition (18). Accordingly, the function $\psi_{a}(x)$ defined by (17) may be considered as a function depending on an arbitrary function 
$\varphi_{a}(x)$ given and continuous in the interval $\left\langle x_{0}, x_{1}\right)$ and fulfilling condition (19). Formula (16) then gives the solution of equation (1) continuous in the interval $(a, b)$ and depending on an arbitrary function, which completes the proof.

\section{References}

[1] J. Kordylewski and M. Kuczma, On some linear functional equations II, Ann. Polon. Math. 11 (1962), p. 203-207.

[2] M. Kuczma, Functional equations in a single variable, Warszawa 1968.

[3] - and P. Vopénka, On the functional equation $\lambda[f(x)] \lambda(x)+A(x) \lambda(x)+B(x)$ $=0$, Annales Universitaties Scientiarum Budapestinensis, tomus III-IV (1960. 1961), p. $123-133$.

Reģu par la Rédaction le 10.12. 1969 